\title{
A REPRESENTAÇÃO SOCIAL DE FAMILIARES NOS CENTROS DE ATENÇÃO PSICOSSOCIAL
}

\author{
Social representation of family members in psychosocial care center \\ La representación social de la familia en centros de atencion psicosocial
}

Dulcian Medeiros de Azevedo

Francisco Arnoldo Nunes de Miranda²

\section{RESUMO}

Os Centros de Atenção Psicossocial (CAPS) estão previstos como as portas de entrada e regulação em saúde mental, e foram criados na intenção de substituir as internações nos manicômios pelo atendimento aberto na comunidade. Objetivou-se apreender as representações sociais dos familiares de usuários dos CAPS do Município de Natal-RN, a respeito de sua participação nas atividades desses serviços. Pesquisa descritiva, com abordagem qualitativa, desenvolvida com 28 familiares por meio de entrevista semiestruturada, entre agosto e setembro de 2007. Os dados obtidos receberam o suporte informacional do software ALCESTE. Os familiares estruturam sua representação social em face de uma necessidade e esperança por mudanças no tratamento de saúde de seus familiares, cristalizadas pela tomada de posicionamento e comportamento, amparados na presença do espaço ambiental dos CAPS. A participação familiar ainda não reúne, nos cenários investigados, condições para promover a inserção do familiar, pois a presença física constatada ainda não é o bastante.

Palavras-chave: Psicologia Social. Serviços de Saúde Mental. Família. Enfermagem Psiquiátrica. Transtornos Relacionados ao Uso de Substâncias.

\begin{abstract}
The Psychosocial Care Centers (CAPS) are foreseen as the ports of entry and regulation in mental health, and were created with the intention of replacing the hospitalization in asylum for the community care service. The objective was to understand the social representations of family members of the CAPS users from Natal-RN, about their participation in the activities of these services. Descriptive research with qualitative approach, developed with 28 family members, through semi-structured interviews between August and September 2007. The data received informational support of ALCESTE software. The family members build their social representation in the face of a need for change and hope in the health care of their relatives, crystallized by taking and positioning behavior, supported in the presence of the environmental space of CAPS. The family participation still does not gather, in the scenarios investigated, conditions to promote the inclusion of their relative, once the physical established presence is not yet enough.
\end{abstract}

Keywords: Psychology, Social. Mental Health Services. Family. Psychiatric Nursing. Substance-Related Disorders.

\section{Resumen}

Los Centros de Atención Psicosocial (CAPS) están previstos como las puertas de entrada y de regulación en materia de salud mental, y fueron creados con la intención de sustituir las internaciones en hospitales psiquiátricos para la atención abierta a la comunidad. Nuestro objetivo es comprender las representaciones sociales de los familiares de los usuarios de un CAPS de la ciudad de Natal-RN, sobre su participación en las actividades de estos servicios. Estudio descriptivo, cualitativo, desarrollado con 28 miembros de las familias, a través de entrevistas seme-estructuradas entre agosto y septiembre de 2007. Los datos obtenidos recibieron soporte del software ALCESTE. Las familias estructuraran su representación social con base en una necesidad y esperanza de cambio en el cuidado de la salud de sus parientes, cristalizada mediante la adopción de la posición y el comportamiento, con el apoyo de la presencia del espacio ambiental del CAPS. En las situaciones investigadas, la participación de la familia no cumple las condiciones para promover la inclusión del usuario, ya que la presencia física no existe aún suficiente.

Palabras claves: Psicología Social. Servicios de Salud Mental. Familia. Enfermería Psiquiátrica. Trastornos Relacionados con Substancias.

'Enfermeiro. Mestre em Enfermagem (PGENF-UFRN) e Doutorando em Ciências da Saúde (PPGCSa-UFRN). Professor Assistente Il do Curso de Graduação em Enfermagem (UERN), Campus do Seridó. Líder do Grupo de Pesquisa "A enfermagem no processo saúde-doença individual/coletiva, na educação em saúde e na assistência/gerência de serviços de saúde". Caicó-RN. Brasil. E-mail: professordulcian@gmail.com Doutor em Enfermagem Psiquiátrica (EERP/USP). Professor Adjunto III do Departamento de Enfermagem (Graduação e Pós-Graduação - UFRN), Campus Central. Líder do Grupo de Pesquisa "Ações promocionais e de atenção a grupos humanos em Saúde Mental e Saúde Coletiva". Natal-RN. Brasil E-mail: farnoldo@gmail.com 


\section{INTRODUÇ̃̃O}

Os Centros de Atenção Psicossocial (CAPS) estão previstos como as portas de entrada e regulação em saúde mental no Sistema Único de Saúde (SUS) e foram criados na intenção de substituir as internações nos manicômios pelo atendimento aberto na comunidade. Têm por finalidade fornecer atendimento à população em área adstrita, com acompanhamento clínico e reinserção social dos usuários pelo acesso ao trabalho, lazer, exercício dos direitos civis, for talecimento dos laços familiares e comunitários. ${ }^{1}$ Estes serviços de saúde mental devem estar circunscritos no espaço de convívio social (família, escola, trabalho, igreja, associações) dos usuários atendidos, objetivando o resgate ou descoberta das potencialidades dos recursos comunitários no entorno.

0 CAPS tem demonstrado efetividade na substituição da internação hospitalar, por um cuidado que não afasta os usuários de suas famílias e da comunidade, capaz de envolver familiares no atendimento, com acolhimento e humanização, ajudando na reabilitação psicossocial. ${ }^{2}$

A exemplo dos CAPS, os serviços substitutivos, enquanto integrantes do programa de atenção à saúde mental alicerçado nos pressupostos da Reforma Psiquiátrica, buscam recolocar a unidade familiar em uma posição de responsabilidade pelo cuidado de seus membros e torná-la agente de transformações nos diversos cenários assistenciais, destacando-se a coparticipação e corresponsabilidade no cuidado a pessoa com sofrimento psíquico. $^{3-5}$

Em alguns países europeus, a partir da década de 1960, e em 1980 no Brasil, a inclusão da família no tratamento à saúde mental passou a ser discutida no âmbito político e entre os profissionais da área. ${ }^{5,6} \mathrm{~A}$ família é convocada a participar efetivamente do processo de reconstrução da dignidade da pessoa com sofrimento psíquico, ocupando lugar privilegiado nas discussões das políticas públicas em saúde mental diante da Reforma., ${ }^{3,5}$

Dessa forma, o comprometimento da família direcionado ao cuidado do portador de transtorno mental passa a exigir uma nova organização familiar e aquisição de habilidades capazes de desarticular o cotidiano e funcionamento deste núcleo. Da mesma forma, o familiar pode se tornar um parceiro da equipe de saúde no cuidado ao usuário, sendo facilitador nas ações de promoção da saúde mental e de inserção do indivíduo na comunidade. ${ }^{2}$

Inicialmente, a ideia de trabalhar com familiares de usuários de serviços de saúde mental surgiu ainda na graduação, a partir da vivência no desenvolvimento de práticas extracurriculares em Saúde Mental e Enfermagem Psiquiátrica, em um manicômio. Diariamente, era constatada a ausência de informações direcionadas aos familiares sobre o transtorno mental e os cuidados ou manejo após a alta.
Aliado a isso, verificava-se a insuficiência de estratégias psicoterapêuticas que envolvessem diretamente o familiar no tratamento do doente no hospital.

Anos mais tarde, ocorreu uma nova vivência em um serviço de saúde mental, agora um CAPS, onde foi permitida a colaboração na execução e na organização do "Domingo no CAPS", projeto que transpunha os muros do serviço chegando à rua, ao exibir os resultados das atividades $\mathrm{e}$ oficinas terapêuticas realizadas. Este contato levou à percepção e conhecimento de uma dinâmica diferente daquela experimentada no hospital, na qual a família deixou de ser uma mera expectadora do tratamento ou, simplesmente, aquela que levava e buscava o usuário, para participar efetivamente no tratamento e no serviço.

Trazer a família para o serviço de saúde mental e ainda fazer dessa um agente ativo de participação, e, portanto, de transformação, representa para os profissionais de saúde um desafio e, ao mesmo tempo, uma mudança de práticas terapêuticas. 0 serviço de saúde mental e 0 profissional passam então a alocar esforços não mais no atendimento individual do doente, destacando o transtorno mental como único foco, mas na coletividade de seus relacionamentos afetivos, sociais, e em especial familiares.

Reconhecendo a família e o contexto social no campo da saúde mental como parte dos recursos que a enfermagem dispõe para a adoção de intervenções profissionais, surgiu a inquietação de como seria investigar a participação do familiar junto à dinâmica de funcionamento das atividades propostas pelos CAPS.

Como estratégia e suporte teórico-metodológico, em reposta a essa inquietação, utilizaram-se a Teoria das Representações Sociais (TRS) e a Teoria do Núcleo Central (TNC), sendo a primeira entendida como "uma modalidade de conhecimento particular que tem por função a elaboração de comportamentos e a comunicação entre indivíduos", em que se produzem e se determinam comportamentos, definindo a natureza dos estímulos que nos cercam e nos provocam, e o significado das respostas a emitir. $7: 26$

Esta teoria seria capaz de proporcionar a compreensão da prática do enfermeiro na interface da qualidade e da quantidade de atividades e procedimentos teórico-metodológicos, voltados ao atendimento das demandas dos usuários dos serviços de saúde mental e psiquiátricos. Nesse sentido, se poderá fornecer subsídios para a reflexão e orientação prática dos profissionais de saúde, especialmente os da enfermagem, atuantes nos CAPS II Leste e Oeste, CAPSad Leste e Norte do município de Natal, escolhidos como campos de pesquisa.

Diante do exposto, o objetivo deste estudo foi apreender as representações sociais dos familiares de usuários dos Centros de Atenção Psicossocial (CAPS) do Município de Natal-RN, a respeito de sua participação nas atividades desses serviços. 


\section{MÉTODOS}

Pesquisa descritiva, com abordagem qualitativa. Os locais de pesquisa foram os CAPS II Leste e Oeste, e CAPSad Leste e Norte, serviços de saúde mental pertencentes à Secretaria Municipal de Saúde de Natal-RN, localizados nos distritos sanitários Leste, Oeste e Norte. A população foi constituída pelos familiares dos usuários atendidos nestes serviços, aproximadamente 70, que participavam das atividades da agenda terapêutica e serviços oferecidos, através dos grupos de família, das reuniões de familiares e das assembleias de usuários, técnicos e familiares, dos passeios e das festividades.

Os critérios de inclusão dos sujeitos foram: a participação regular do familiar em uma das estratégias de participação desenvolvidas nos CAPS há pelo menos três meses; e ter parente usuário em tratamento no CAPS. Após esclarecimento sobre os objetivos e finalidades da pesquisa, os sujeitos assinaram o Termo de Consentimento Livre e Esclarecido (TCLE). A pesquisa foi aprovada pelo Comitê de Ética em Pesquisa da Universidade Federal do Rio Grande do Norte (CEP-UFRN), parecer 118/2007.

A entrevista semiestruturada foi 0 instrumento de pesquisa utilizado, e a coleta de dados ocorreu entre os meses de agosto e novembro de 2007, sendo as falas dos 28 familiares entrevistados gravadas em áudio digital (Aparelho MP4 Foston/ FS-58), sete em cada serviço pesquisado.

Os dados obtidos na entrevista receberam o suporte informacional do software ALCESTE (Analyse Lexicale par
Contexte d'un Ensemble de Segments de Texte), considerado pioneiro no uso da informática na análise de conteúdo, criado na França na década de $1970 .{ }^{8}$ Em seguida, o material discursivo foi analisado mediante o recurso técnico-metodológico da TRS ${ }^{7}$ e da TNC. ${ }^{9}$

Este artigo integra um estudo inédito ${ }^{10}$ acerca da participação familiar nos CAPS do município de Natal-RN, em meio à organização e implementação da rede de saúde mental.

\section{RESULTADOS E DISCUSSÕES}

Os familiares entrevistados eram em sua maioria mulheres (85,7\%), casadas $(71,5 \%)$, com idade superior a 50 anos $(64,0 \%)$. Tais cifras são comuns na identificação dessas donas de casa, idosas, provedoras da saúde familiar e cuidadoras, como as responsáveis pelo equilíbrio do lar e pela procura de serviços de saúde na família, ocupando as esferas da vida pública e privada.

0 aparecimento do gênero feminino no tratamento e acompanhamento da pessoa com sofrimento psíquico ou usuária de drogas nos serviços de saúde mental é um achado natural nos vários estudos correlatos sobre participação familiar. ${ }^{11-13}$

0 trabalho executado pelo ALCESTE teve um aproveitamento de $68,93 \%$, com geração de seis classes e 488 formas de unidades de contexto elementares (UCEs) analisadas. Como artifício para a construção dos resultados e a compreensão na leitura, foi criado um sistema de correspondência nominal entre as classes obtidas pelo ALCESTE e as categorias elaboradas pelos pesquisadores mediante os significados dos achados discursivos (Quadro 1).

Quadro 1 - Correspondência entre a Classificação Hierárquica e o Sistema Categorial

\begin{tabular}{|l|c|}
\hline Categoria 1: Tratamento - Melhoras e Expectativas & Classe 4 \\
\hline Categoria 2: Convivência - Usuários Antes e Depois & Classe 1 \\
\hline Categoria 3: Atividades - Importância, Contradições e Sugestões & Classe 2 \\
\hline Categoria 4: Orientações - Psicofarmacologia e Medicalização & Classe 3 \\
\hline Categoria 5: Família - Participação e Atividades & Classe 6 \\
\hline $\begin{array}{l}\text { Categoria 6: Condições Terapêuticas - Agradecimentos, Sugestões e } \\
\text { Vulnerabilidade }\end{array}$ & Classe 5 \\
\hline
\end{tabular}

É importante esclarecer que a utilização da TNC neste estudo, e em outro qualquer, não tem o valor de complementaridade à TRS, pois não objetiva outra coisa senão focalizar a atenção sobre os próprios fenômenos do campo representacional, dando pistas ao que inicialmente Moscovici chamou de núcleo figurativo. ${ }^{14}$

A organização de uma ou mais representações sociais apresenta uma característica peculiar que é a de ser organizada em torno de um núcleo central, com dois ou mais elementos que dão significado à representação, em uma perspectiva de abordagem estrutural do que é representado. ${ }^{9}$

A Figura 1 exibe a formação do campo representacional acerca da participação familiar nos CAPS de Natal. Ela é constituída pelas linhas $x$ e y, horizontal e vertical, que compõem quatro partes iguais de uma mesma imagem e uma distribuição espacial das categorias em seus respectivos quadrantes: superior direito e superior esquerdo (QSD e QSE), inferior direito e inferior esquerdo (QID e QIE). 
Figura 1 - Esquema Figurativo e Conformação dos Quadrantes perante o Núcleo Central das Representações Sociais dos familiares sobre a participação nos CAPS. Centros de Atenção Psicossocial de Natal-RN, 2007

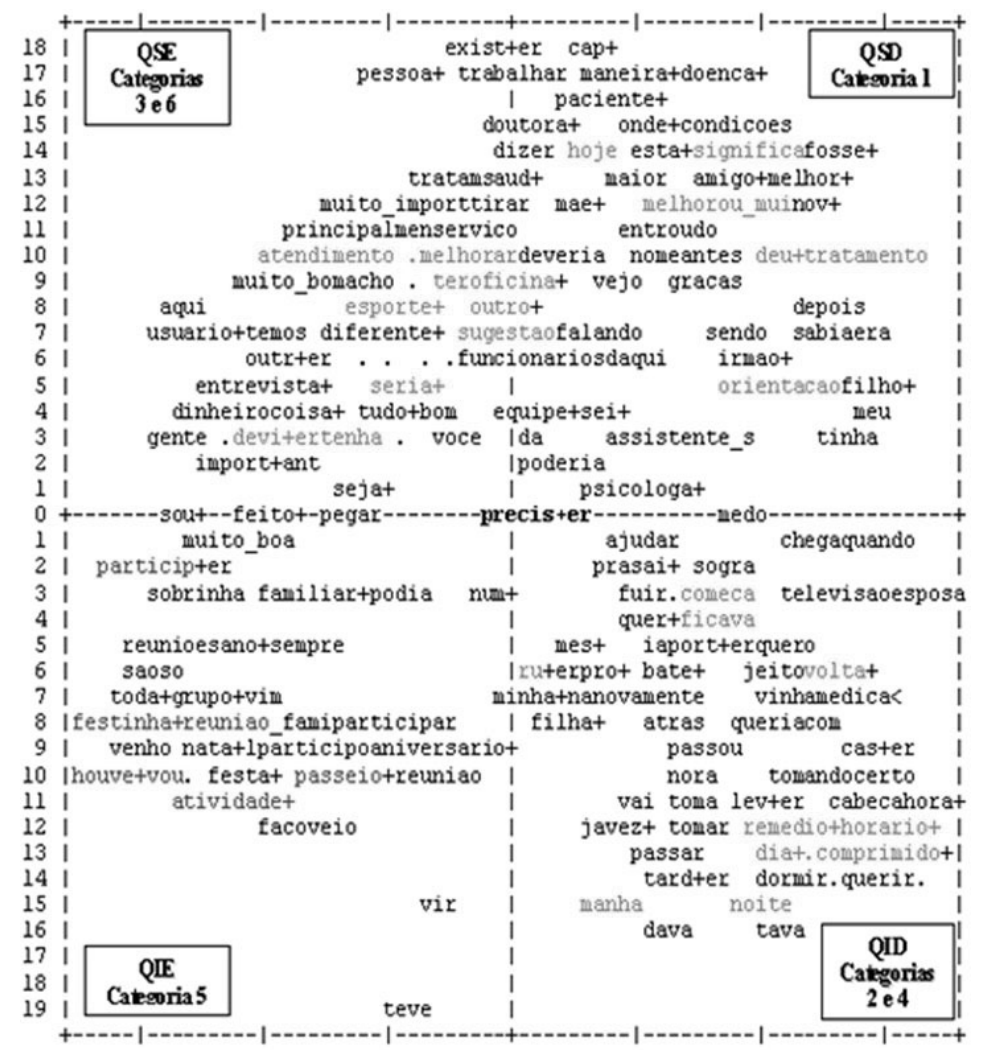

0 QSD é composto pelas unidades semânticas mais significantes, distribuídas pelo ALCESTE, relacionadas às mudanças positivas do processo saúde-doença mental dos usuários a partir do tratamento experimentado nos CAPS. 0 seu conjunto semântico está localizado nas UCEs pertencentes à Categoria 1, "Tratamento - Melhoras e Expectativas".

0 QSI exibe, predominantemente, os termos pronunciados pelos familiares a respeito de sugestões sobre o desenvolvimento e existência de atividades terapêuticas direcionadas aos usuários, especialmente as oficinas, como uma tentativa de aumentar o leque de possibilidades terapêuticas, dando suporte ao ideal de melhora exibido no QSD. Esse quadrante é constituído pelas Categorias 3 e 6, denominadas "Atividades - Importância, Contradições e Sugestões" e "Condições Terapêuticas - Agradecimentos, Sugestões e Vulnerabilidade", respectivamente.

No QID estão presentes as raízes semânticas relacionadas a vivências familiares no acompanhamento e trajetória do transtorno mental ou do uso de drogas dos usuários, experienciadas em um contexto externo ao CAPS, além de trazer um reforço à medicalização enquanto ferramenta no tratamento atual. As Categorias 2 e 4, intituladas "Convivência - Usuário Antes e Depois", e "Orientações - Psicofarmacologia e Medicalização", acomodaram-se nesse quadrante.

Já o QIE registra as formas participativas ou situações em que ocorre a presença dos familiares nos CAPS investigados. A reunião, o grupo e a festa são exemplos de raízes semânticas que correspondem a essa forma participativa, localizados nas UCEs da Categoria 5, "Família - Participação e Atividades".

Mediante esta linha de análise, os familiares estruturam sua representação social em face da necessidade e esperança por mudanças no tratamento de saúde de seus familiares, cristalizada pela tomada de posicionamento e comportamento amparados na presença do espaço ambiental dos CAPS, mesmo que em alguns momentos essa seja estritamente física, fugaz e incipiente, ou não inclusiva.

Estes familiares carregam consigo experiências diversas de sofrimento, angústias e maus tratos vivenciados a partir do transtorno mental ou do uso de drogas de seu familiar. Seja nos cenários de tratamento, seja no lar ou em ambientes externos a esse, os familiares, por não desejarem mais que se repitam, veem nos CAPS essa possibilidade em virtude dos resultados positivos alcançados pelo tratamento.

Eles buscam e acreditam que é preciso mudar, "botar pra fora" e mobilizar caminhos e ações, para que haja a continuidade das atividades terapêuticas e superação das inconsistências detectadas, a exemplo da ampliação estrutural dos serviços e seu funcionamento nos três turnos, do aumento ou possibilidade de participação nos passeios, da aprendizagem de alguma atividade profissional para inserção no mercado de trabalho e da geração de renda.

Defende-se que convocar a família para assumir a responsabilidade do cuidado do usuário conjuntamente com a equipe e oferecer espaços de participação exige mais do que 
um simples redirecionamento de práticas. Exige também comprometimento e responsabilidade pela construção de um cuidado que, obrigatoriamente, deve ser coletivo, na busca da promoção e manutenção da autonomia do usuário, reconquistando sua cidadania e seu espaço na sociedade. ${ }^{15}$

A ancoragem estabelece-se no desejo por mudanças relacionadas ao aumento e ajustes das oficinas terapêuticas, na oferta de outras atividades terapêuticas que proporcionem maior espaço, que "cheguem junto", mobilizando usuários, familiares técnicos e comunidade. Essas mudanças objetivam o reforço e a estabilidade das melhorias nas condições de vida e saúde vivenciadas atualmente através do tratamento.

Outro estudo ${ }^{15}$ identificou as oficinas terapêuticas, 0 atendimento individual e a visita domiciliar como estratégias de participação dos familiares em um CAPS II, a partir de entrevistas com profissionais do serviço. Além disso, as festas comemorativas foram instrumentos facilitadores no trabalho de inserção dos familiares, pois, além de contribuírem no conhecimento dos familiares, estreitaram os laços e os convidaram a participar de outras atividades.

0 núcleo semântico "precis+er", destacado no centro da Figura 1, manifestou o maior poder de aproximação simbólica a essa representação social, dada a sua presença significativa em quatro categorias, tanto do ponto de vista quantitativo, na frequência e qui-quadrado observados, como também sob o enfoque qualitativo de significância discursiva encontrada nas UCEs. A Categoria 1 possuiu uma frequência de $100 \%$ e quiquadrado 8,83; as Categorias 2 e 3, frequência de $50 \%$, quiquadrado 2,07 e 3,18, respectivamente; e a Categoria 6, frequência de 33,3 e qui-quadrado 2,98.

A representação social elaborada pelos familiares dos CAPS circula e reúne experiências, vocabulários, conceitos e discursos diversos, ${ }^{7}$ circunscrevendo a experiência do adoecimento e seu curso, a convivência no tratamento de seu familiar antes ou depois dos CAPS, a medicalização experimentada e difundida como imprescindível nesses serviços e a esperança de que ainda há muito a ser feito, modificado, aperfeiçoado nas atividades propostas pelos CAPS investigados.

Tais características, acontecimentos e vivências concretizam, regulam e suportam a crença dos familiares de que os CAPS investigados reúnem condições favoráveis capazes de efetuar melhorias no processo saúde-doença mental de seu familiar usuário.

Portanto, a representação social dos familiares sobre a participação nos CAPS é considerada polêmica, pois está inserida em um contexto recente de práticas terapêuticas em saúde mental, amparadas no paradigma psicossocial, sem jamais desmerecer as influências da psiquiatria clássica e do paradigma da doença mental vivido durante séculos no imaginário social e, por vezes, sentido de perto pela maioria dos familiares no "tratamento" com suporte manicomial.

No que se refere ao campo representacional, o núcleo centra/correspondeu exatamente a uma expectativa ou melhoria concreta nas condições de vida e saúde do usuário perante seu sofrimento mental ou uso de substâncias psicoativas, proporcionada pelo tratamento e "suporte familiar" oferecido nos CAPS.

Seus núcleos semânticos destacados no QSD evidenciam o sistema nuclear, unificando e estabilizando a representação. Entretanto, alertam ${ }^{9: 31}$ que a centralidade de um elemento não pode ser atribuída somente por critérios quantitativos, pois "o núcleo central possui, antes de tudo, uma dimensão qualitativa". Coincidentemente, nesse estudo, a Categoria 1 foi a que respondeu pelo maior número de UCEs.

Os elementos periféricos foram estruturados de maneira a reforçar ainda mais o seu núcleo central, visualizados no QSE e QID. 0 sistema periférico concebido ainda concretiza o núcleo central na tomada de decisões, condutas ou posturas dos familiares dos CAPS, sendo sensivel e determinado pelas características do contexto imediato, que é a própria participação nos serviços de saúde mental pesquisados.

Mais do que isso, os elementos periféricos regulam e adaptam o núcleo central aos constrangimentos e às características da situação real experimentada pelo grupo. ${ }^{9}$ São exemplos desses elementos as oficinas que em alguns momentos não funcionam, os passeios que não atendem a todos e as assembleias que não encaminham nem solucionam os problemas que se apresentam.

A representação social aqui elaborada não é, nem poderia ser, uma simples ordenação ou justaposição da categorização proposta na análise. É necessário ver a forma como esse sistema categorial, uma verdadeira rede de sentidos, articula-se, ordena-se e configura-se em um campo bem estruturado ${ }^{16}$, ainda que suas facetas estejam entremeadas, implícitas e mascaradas.

Por sua vez, o QIE intermedeia o campo representacional através de conteúdos semânticos mais flexíveis que interagem com o sistema central e o periférico, constituindo os elementos intermediários. Os momentos de participação familiar, nos CAPS identificados nesse quadrante, possuem características variadas de pressão, inferência e, especialmente, dispersão da informação, ${ }^{17}$ pois é nesse ambiente que os familiares assimilam, trocam, absorvem, (des)constroem conceitos e saberes sobre o transtorno mental e uso de substâncias psicoativas.

A representação social elaborada demarca um campo figurativo, tendo por base a forma como o sujeito hierarquiza e coordena os significados e as atitudes confrontados com o seu levantamento sistemático e 0 repertório simbólico a respeito do objeto desta mesma representação. ${ }^{7}$ Portanto, não existe uma realidade objetiva a princípio, pois toda realidade é representada, reapropriada no plano individual ou grupal, reconstruída 
no sistema cognitivo, associada ao sistema de valores e dependente da historicidade e do contexto social e ideológico que os cercam. ${ }^{9}$

Dessa forma, as representações sociais têm um papel fundamental na dinâmica das relações sociais e nas práticas, por isso respondem por quatro funções espećificas: permitem compreender e explicar a realidade; definem a identidade, permitindo a proteção da especificidade dos grupos; guiam o comportamento e as práticas sociais; e, por fim, admitem, inicialmente, a justificativa das tomadas de posição e comportamentos. ${ }^{7,9}$ Ao término da análise, serão identificados, no conjunto discursivo dos familiares, os depoimentos que ilustram e mais se aproximam das funções estabelecidas pelo sistema representacional. ${ }^{7,9}$

A compreensão e a explicação da realidade são observadas nesses trechos das entrevistas:

É como uma mãe [o CAPS]. Uma mãe, que cuida bem dos seus pacientes (...) tem me ajudado muito, (...) eu não sabia lidar com a situação (Filha, CAPS II Oeste, E-11).

A função identitária do grupo define a identificação e proteção da especificidade do grupo:

CAPS, pra mim, posso dizer que é uma casa. $\dot{E}$ onde me sinto acolhida. É onde eu encontrei a orientação pra ver a dependência do meu filho (Mãe, CAPSad Norte, E-03).

A função orientadora conduz os comportamentos e as práticas:

E também pra mim poder ajudar ele [esposo], como a gente tem as reuniões, que é pra gente saber lidar com eles. Que antes a gente não sabia, brigava, achava que era safadeza, mas não! Aí depois do tratamento, (...) vimos que é uma doença, e temos que ajudar eles (Esposa, CAPSad Leste, E-09).

Para finalizar, a função justificadora permite o posicionamento e o desenvolvimento do comportamento:

A gente não tem mais medo de cuidar dele [irmão] porque a gente foi orientado. (...) Nós aprendemos a fazer combinados [risos]. Isso é muito interessante! [...] antes como a gente temia, não conhecia, achava que ele podia se comportar mal, evitava sair com ele. Mas, hoje não. A gente fica chamando pra incentivar ele a sair da rotina (Irmã, CAPS II Oeste, E-10).

\section{CONCLUSÕES}

Afinalização desta pesquisa permitiu aos pesquisadores vivenciar possibilidades infinitas de aprendizado na interação com grupos distintos, porém com traços comuns de identificação e autenticidade, de pessoas, familiares, usuários e profissionais de saúde mental nos serviços pesquisados e na sala de aula enquanto estudante de pós-graduação.

A interface entre conhecimento científico e senso comum, a partir da aproximação dos gestores nos serviços, dos familiares, dos usuários, da interação discursiva no diálogo com esses atores e das falas, expressões analisadas e contidas nas entrevistas, reforça o campo fértil das relações humanas, em seus modos de pensar, desejar e agir, mas também em afetar e em dissimular, características inerentes ao homem.

Através da presença nos serviços e da convivência no domicílio, os familiares identificaram que o tratamento recebido nos CAPS favorece melhoras substanciais nas condições de vida e de saúde de seu familiar usuário e nas relações familiares dentro e fora do lar, ainda que seja detectada no desenvolvimento das atividades terapêuticas a necessidade de ajustes, de aumento da oferta e um maior envolvimento da equipe técnica.

Apesar dessas formas participativas serem, reconhecidamente, importantes no acompanhamento e tratamento da pessoa com sofrimento psíquico e usuário de drogas, elas ainda não reúnem, nos cenários investigados, condições para promover a inserção do familiar, pois a presença física constatada ainda não é o bastante.

Esta inserção requerida, e sob um ponto de vista emancipador, é capaz de suscitar no sujeito o desejo da autonomia, da iniciativa, do crescimento individual e coletivo e de um envolvimento mais próximo e ativo nas atividades terapêuticas, nas oficinas e nas discussões. Infelizmente, esse movimento interacional nos familiares entrevistados não foi observado conjuntamente.

A pesquisa ora apresentada não se constituiu, pelo menos no que fora idealizado inicialmente, em um mecanismo de avaliação das condições de oferta e qualidade dos serviços prestados na rede municipal de saúde mental de Natal-RN. Entretanto, a satisfação dos familiares nas melhoras de saúde alcançadas e os acertos do serviço ofertado, bem como as incongruências, desajustes, na perspectiva do discurso familiar, estabelecem uma de suas contribuições e demonstram sua relevância no cenário da política municipal de saúde mental.

Partindo-se do pressuposto que um estudo não se finaliza em si mesmo, mas abre outras possibilidades, aflora outros fenômenos e detecta pontos de fuga, surgem a necessidade e a curiosidade de fazer o caminho inverso, ou seja, apreender os significados impostos pelos profissionais de saúde ao desejarem promover a participação e a inserção familiar nos CAPS investigados. 


\section{REFERÊNCIAS}

1.Ministério da Saúde (BR). Saúde mental no SUS: acesso ao tratamento e mudança do modelo de atenção. Relatório de gestão: 2003-2006. Brasília (DF);2007.

2.Schrank G, Olschowsky A. 0 centro de atenção psicossocial e estratégias para inserção da família. Rev Esc Enferm USP. 2008; 42(1): 127-34.

3.Moreno V, Alencastre MB. A família do portador de sofrimento psíquico e os serviços de saúde mental: estudo de caso. Acta Sci, Health Sci. 2004 jan/jun; 26(1): 175-81.

4.Azevedo DM, Miranda FAN. The family and substitute services in mental health: a clipping of the brazilian literature in nursing. Rev Enferm UFPE On line [periódico on-line]. 2009; [citado 2009 maio 04]; 3(1): 93-98. Disponivel em: http://www.ufpe.br/revistaenfermagem/index.php/ enfermagem/article/view/257/295

5.Azevedo DM, Miranda FAN. Práticas profissionais e tratamento ofertado nos CAPSad do município de Natal-RN: com a palavra a família. Esc Anna Nery. 2010 jan/mar; 14(1): 56-63.

6.Souza RC, Pereira MAO, Scatena MCM. Família e transformação da atenção psiquiátrica: olhares que se (des)encontram. Rev Gaucha Enferm. 2002; 23(2): 68-80.

7.Moscovici S. A representação social da psicanálise. Rio de Janeiro: Zahar; 1978.

8.Camargo VB. ALCESTE: um programa informático de análise quantitativa de dados textuais. In: Moreira ASP, organizador. Perspectivas teóricometodológicas em representações sociais. João Pessoa: UFPB/Ed Universitária; 2005. p. 511-39.

9.Abric JC. A abordagem estrutural das representações sociais. In: Moreira ASP,Oliveira DC, organizadores. Estudos interdisciplinares de representação social. $2^{a}$ ed. Goiânia: AB Ed ; 2000. p. 27-38.

10.Azevedo DM. Estudo representacional da participação familiar nas atividades dos centros de atenção psicossocial no município de NatalRN. [dissertação]. Natal (RN): Departamento de Enfermagem, Universidade Federal do Rio Grande do Norte; 2008.

11.Vargas NIT, Zago MMF. El sufrimiento de la esposa em la convivencia com el consumidor de bebida alcoólicas. Rev Latino-am Enfermagem, 2005 nov/dez; 13(n esp): 806-12.

12.Filizola CLA, Perón CJ, Nascimento MMA, Pavarini SCI, Petrilli Filho JF. Compreendendo o alcoolismo na família. Esc Anna Nery. 2006 dez; 10(4): 660-70.

13.Azevedo DM, Gaudêncio MMP. A esquizofrenia sob a ótica familiar: discurso dos cuidadores. Nurs 2007 ago; 111(10): 366-71.

14.Sá CP. Núcleo central das representações sociais. $2^{\mathrm{a}}$ ed. Rio de Janeiro: Vozes; 2002.

15.Schrank G. 0 centro de atenção psicossocial e a inserção da família [dissertação]. Porto Alegre: Escola de Enfermagem, Universidade Federal do Rio Grande do Sul; 2006.
16.Arruda A. Despertando do pesadelo: a interpretação. In: Moreira ASP et al, organizadores. Perspectivas teórico-metodológicas em representações sociais. João Pessoa: Ed Universitária/UFPB; 2005. p. 229-58.

17.Wagner W, Elejabarrieta F. Representaciones sociales. In: Morales F, organizador. Psicología social. Madrid: Mcgraw-Hill; 1994. p. 815-39. 